\title{
JN

\section{CALL BROADCASTING AND AUTOMATED RECORDERS AS TOOLS FOR ANURAN SURVEYS IN A SUBARCTIC TUNDRA LANDSCAPE}

\author{
R. NICHOLAS MANNAN ${ }^{1}$, GAD PERRY ${ }^{1,2}$, DAVID E. ANDERSEN ${ }^{3}$, AND CLINT W. BOAL ${ }^{4}$ \\ ${ }^{1}$ Department of Natural Resources Management, Texas Tech University, Lubbock, Texas, 79409, USA \\ 2 Corresponding author. E-mail: Gad.Perry@ttu.edu \\ 3 U.S. Geological Survey, Minnesota Cooperative Fish and Wildlife Research Unit, Department of Fisher- \\ ies, Wildlife, and Conservation Biology, University of Minnesota, St. Paul, Minnesota, 55108, USA \\ 4 U.S. Geological Survey, Texas Cooperative Fish and Wildlife Research Unit Department of Natural \\ Resources Management, Texas Tech University, Lubbock, Texas, 79409, USA
}

\begin{abstract}
Relatively little is known about population ecology of anurans in arctic and subarctic tundra regions, in part because it is difficult to survey anurans in these landscapes. Anuran survey protocols developed for temperate regions have limited applicability in arctic and subarctic tundra landscapes, which may lack roads and vehicle access, and experience variable and inclement weather during short anuran breeding seasons. To evaluate approaches to address some of the limitations of surveying anurans in tundra landscapes, we assessed the effectiveness of using breeding call broadcasts to increase detection of Boreal Chorus Frogs (Pseudacris maculata) and Wood Frogs (Lithobates sylvaticus) near Cape Churchill, Manitoba, Canada. We also evaluated how counts of anurans derived from automated audio recorders compared with those obtained simultaneously by observers. We detected on average 0.4 additional Wood Frogs per survey when we broadcasted calls $(\bar{x}=0.82, S D=1.38)$, an increase of $>40 \%$ compared to surveys without broadcasts $(\bar{X}=1.24, S D=1.51$; Wilcoxon test; $Z=2.73$, $P=0.006)$. In contrast, broadcasting Boreal Chorus Frog calls did not increase the number of chorus frog detections (Wilcoxon test; $Z<0.001, P>0.90$ ). Detections of Wood Frogs in a $100-m$ radius were lower via automated recorders $(\bar{X}=0.60, S D=0.87 \mathrm{SD})$ than by observers during simultaneous surveys $(\bar{X}=0.96, S D=1.27 Z=2.07, P=0.038)$, but those of Boreal Chorus Frogs were not different ( $\bar{X}=1.72, S D=1.31 ; \bar{x}=1.44, S D=1.5 ; Z=1.55, P>0.121)$. Our results suggest that broadcasting calls can increase detection of Wood Frogs, and that automated recorders are useful in detecting both Wood Frogs and Boreal Chorus Fogs in arctic and subarctic tundra landscapes.
\end{abstract}

Key words: Anuran surveys, automated recorder, Boreal Chorus Frogs, Lithobates sylvaticus, Pseudacris maculata, tundra, Wood Frogs

\section{INTRODUCTION}

Established survey protocols for anurans include roadside surveys, listening stations, repeated surveys at selected breeding areas, and counts of egg masses (Heyer et al. 1994, Crouch and Paton 2000, Gerhardt et al. 2000, MacKenzie et al. 2002) and most incorporate detection of breeding calls (e.g., Heyer et al. 1994, Mackenzie et al. 2002). However, applying established anuran survey protocols developed for circumstances common in more temperate latitudes (e.g., extensive road networks, relatively long anuran breeding seasons) to many high-latitude landscapes is not straightforward. In arctic and subarctic tundra landscapes, anuran breeding seasons are relatively short and are often characterized by highly variable and extreme weather conditions. In at least some locations, reproduction occurs during periods that may include risk of encounters with dangerous wildlife such as polar bears (Ursus martimus), requiring added safety precautions and possibly forgoing data collection during periods when calling activity may be highest, such as near dusk. Moreover, survey protocols developed in more accessible landscapes, such as road-side listening surveys and repeated surveys at selected breeding areas (Heyer et al. 1994, Crouch and Paton 2000) are generally not feasible in roadless areas. There are no standard anuran survey protocols for arctic and subarctic tundra landscapes, where relatively few surveys of anurans have been performed and little is known about the 
distribution and population dynamics of anurans.

Near the coast of Hudson Bay in Manitoba, Canada, Boreal Chorus Frogs (Pseudacris maculata) and Wood Frogs (Lithobates sylvaticus) occur across a gradient from coastal tundra through interior sedge meadow-wetland to the tundra-boreal forest interface (Reiter et al. 2008). In this subarctic landscape, freshwater bodies are frozen and snow cover persists for up to 9 months a year, resulting in anuran breeding habitat being available for only a few months annually. During this period, travel is largely restricted to aircraft for longer distances or on foot for shorter distances. For example, Reiter et al. (2008) conducted line-transect surveys for calling Boreal Chorus Frogs and Wood Frogs across this landscape, and used helicopters for transportation among survey sites. Theirs was the first study to assess landscape-scale distribution and habitat associations of these species in a subarctic tundra landscape, but whether sufficient resources, especially helicopter time, are available may limit the broad application of their approach.

Protocols that increase anuran detection may reduce the number of replicate surveys necessary to precisely estimate occupancy (e.g., MacKenzie et al. 2002), and likely increase survey efficiency (i.e., provide more information with the same or less investment of resources). One approach to increasing detection of cryptic species is inducing a response from the animals being surveyed. For example, a variety of bird survey protocols include broadcasting calls to elicit responses from individuals that would have otherwise gone undetected (e.g., Conway and Simon 2003, Andersen 2007). Based on previous studies that used call broadcasts to elicit responses and increase anuran detection (e.g., Wells 1977, Wells and Greer 1981, Gerhardt 1982, Gerhardt et al. 2000), we hypothesized that broadcasting breeding calls of Boreal Chorus Frogs and Wood Frogs could potentially increase detection, and therefore increase survey efficiency. Herein we evaluate whether broadcasting Boreal Chorus Frog and Wood Frog breeding calls increases detection in a subarctic tundra landscape. In addition, because both Boreal Chorus Frogs and Wood Frogs call intermittently throughout the day in our subarctic tundra study area, we hypothesized that automated audio recorders (hereafter automated recorders) may further increase detection (e.g., Peterson and Dorcas 1992, 1994; Dorcas et al. 2010).

\section{METHODS}

Study area - We conducted our study in the vicinity of the Nestor One research camp $\left(58^{\circ} 34^{\prime} \mathrm{N}, 9^{\circ} 11^{\prime} \mathrm{W}\right)$ in Wapusk National Park (WNP) of Canada. WNP is located along the western shore of Hudson Bay, Manitoba, Canada and covers about $11,475 \mathrm{~km}^{2}$. The park lies within the Hudson Bay Lowlands, which are characterized by poorly drained peatlands, with a matrix of small upland ridges and lowland sedge-dominated marshes near the coast (Didiuk and Rusch 1998, Brook 2001). Permafrost underlies the entire region, resulting in most water bodies being $<1 \mathrm{~m}$ deep. Winter temperatures average $-26^{\circ} \mathrm{C}$, and summer temperatures average $11^{\circ} \mathrm{C}$ (http://www.pc.gc. $\mathrm{ca} / \mathrm{pn}-\mathrm{np} / \mathrm{mb} /$ wapusk/visit/visit4_e.asp). The town of Churchill, located about $65 \mathrm{~km}$ northwest of Nestor One and hosting the nearest weather station, receives an average of $436 \mathrm{~mm}$ of precipitation per year (http://www. weatheroffice.gc.ca).

We conducted field work inside two $12.6-\mathrm{km}^{2}$ study plots, situated $8 \mathrm{~km}$ north and south of Nestor One. Both study plots were located in freshwater, sedge-dominated wet meadows. We used ArcGIS version 9.1 (ESRI, Redlands, Calif., USA) to randomly select 60 points in each plot; all points were $>200 \mathrm{~m}$ apart to avoid overlap in detection of calling frogs between adjacent points. We adjusted the location of points to ensure that all were within $50 \mathrm{~m}$ of the nearest water body $>10 \mathrm{~cm}$ deep, because shallower water bodies typically dry up early in the spring, and would therefore not provide suitable breeding sites for anurans. After adjusting point locations, we retained 57 points in the northern plot and 60 points in the southern plot that represented potential anuran breeding sites.

Call-broadcast surveys - Between 30 May and 18 June 2006, we conducted three call-broadcast surveys at approximately 6-day intervals at each potential breeding site $(n=57)$ in our northern plot. Between 31 May and 11 July 2007, we surveyed a subsample $(n=27)$ of the 57 sites in our northern plot, and conducted surveys at the 60 potential breeding sites in our southern study plot. In 2007 we also systematically physically searched both study plots for the presence of anurans. To do this, we repeatedly ( $\geq 2$ times) walked 8 transects located at $1-\mathrm{km}$ intervals spanning each study plot. When we detected anurans, we walked to the site from which anurans were calling and marked the location to facilitate subsequent broadcast surveys. We subsequently conducted broadcast surveys at 11 of these locations and conducted a total of 155 broadcast surveys distributed across breeding sites on our northern and southern study plots. In our statistical analyses, we included data from 99 surveys, consisting of the first Wood Frog, Boreal Chorus Frog, or null broadcast at each of the 47 sites where we detected anurans. We broadcasted calls or applied our null treatment (no broadcast) during 41 surveys at 24 sites where we detected Wood Frogs. Similarly, we broadcasted calls or applied our null treatment during 18 surveys at 16 sites where we detected Boreal Chorus Frogs. We conducted call broadcast surveys when wind speeds were $<10 \mathrm{~km} / \mathrm{h}$.

Each broadcast survey consisted of the same single observer who stood $5 \mathrm{~m}$ from the potential breeding site. We initiated each survey following an initial 1-min period, during which the observer quietly stood at the survey location. Following this initial period, the observer recorded the number and species of anurans detected during

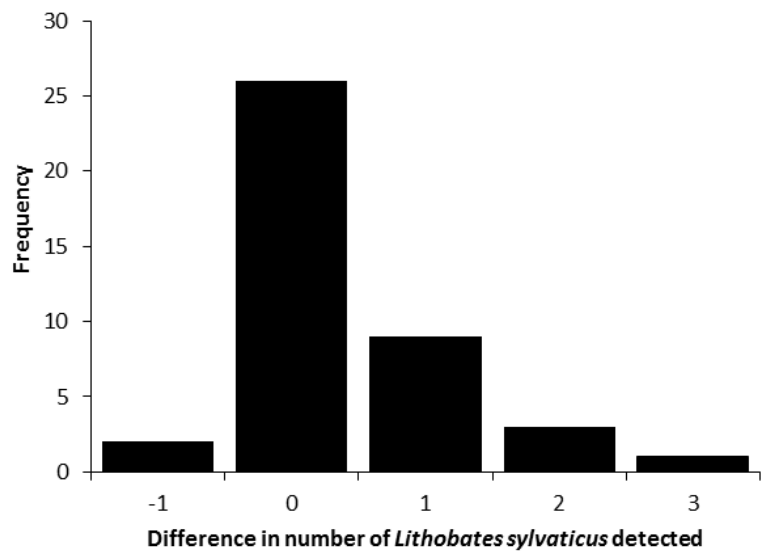

Figure 1. Difference in the numbers of Lithobates sylvaticus detected after versus before broadcasting conspecific calls near Cape Churchill, Manitoba, 2006-2007. This difference represents the increase in the number of $L$. sylvaticus detected following call broadcasts. 
a 3-min listening period. In two cases when anuran calls overlapped, we estimated the minimum number of frogs present. Following the initial 3-min listening period, the observer then randomly offered one of three treatments, regardless of whether frogs had been heard: 1-min broadcast of calls of Boreal Chorus Frogs; 1-min broadcast of calls of Wood Frogs; or 1 min with the absence of any broadcast (hereafter null treatment). During surveys in 2006, we broadcasted anuran calls obtained from the U.S. Geological Survey Amphibian Research and Monitoring Initiative (http://www.umesc.usgs.gov). During surveys in 2007, we broadcasted calls recorded at our study sites. We broadcasted calls using a megaphone (FOXPRO FX3, AllPredatorCalls.com), which we rotated $360^{\circ}$, at an average of $67 \mathrm{~dB}$ (range $=64-71$ ) in 2006 and $78 \mathrm{~dB}$ (range $=71-80$ ) in 2007 (sound pressure levels measured $1 \mathrm{~m}$ from the source; C-weighting; Radio Shack Sound Level Meter 33-2050). We selected these sound pressure levels at which to broadcast calls because they were the highest sound pressure levels attainable with our recordings and broadcast equipment that did not result in obvious distortion. These sound pressure levels are near or slightly below the range of sound pressure levels (72 - $94.5 \mathrm{~dB}$ ) reported by Gerhardt (1975) for 19 species of North American frogs and toads and slightly higher than those of Wood Frog calls we measured opportunistically on our study sites. Following the call broadcast, we conducted another 3-min listening period. In our study area, the density of anurans was relatively low (Reiter et al. 2008, present study), generally allowing us to distinguish individuals and count the number of calling anurans. However, when $\geq 5$ anurans were calling simultaneously, we recorded the minimum discernible number of individuals.

In our analyses, we treated surveys at the 27 potential breeding sites we surveyed on our northern study site in both 2006 and 2007 as independent observations. We included data in analyses from survey locations where we detected a calling anuran at least once during the year each location was surveyed (i.e., we censored locations where we detected no anurans). To avoid pseudoreplication, we restricted our analyses to the first treatment type at each survey location where we detected a calling anuran at least once during the year we surveyed that location (i.e., both the first call broadcast for each species and the first null treatment at each survey location could be included in our sample). To assess whether our initial

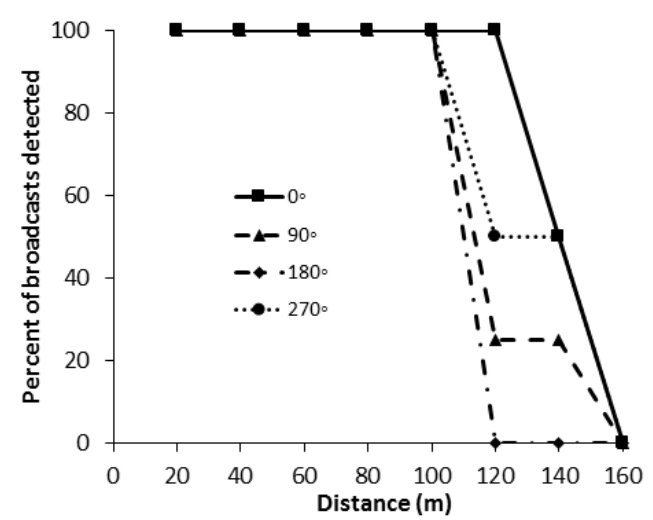

Figure 2. Percent of Lithobates sylvaticus broadcast calls detected on automated recorders as a function of distance in the 4 cardinal directions around recorders near Cape Churchill, Manitoba, 2006-2007. 1-min period was sufficient and to establish a baseline against which to evaluate the effect of broadcasting calls, we compared the number of anurans detected during the initial 1-min period and the null treatment for each location where we applied the null treatment.

To assess whether broadcasting calls elicited calling in Boreal Chorus Frogs and Wood Frogs (and therefore increased detection) we compared the number of anurans of each species detected during the 3 -min periods before and after conspecific-call broadcasts using a Wilcoxon matched-pairs test. To be certain that any differences we observed were in response to call broadcasts and not differences in background call rates at the time of surveys, we also compared the number of both Wood Frogs and Boreal Chorus Frogs detected prior to treatment during surveys where we broadcasted calls and surveys with null treatments using a Mann-Whitney U-test. We used non-parametric tests because our sample sizes were relatively small and our data were not distributed normally (e.g., Figure 1).

Automated recorders - In 2006 and 2007, we placed purpose-built automated recorders (see Mannan 2008 for a detailed description of automated recorder specifications and components) at locations we expected calling anurans to be present for 6-week intervals from late May through early July. Automated recorders consisted of a digital voice recorder, digital timer, microphone, 12volt battery, and a solar panel. We separated recorders from each other by $>200 \mathrm{~m}$ and programmed them to record ambient sounds for 3 min at 1.4-h intervals. We chose a 3-min recording period because most anuran species are detected within the first $3 \mathrm{~min}$ of the start of a survey (Shirose et al. 1997). We listened to recordings during the field season and recorded the number of frogs of each species distinguishable during each 1.4-h interval. Because we did not know the audial sensitivity of automated recorders, we also tested their capability to register broadcasted calls at different distances. We broadcasted Wood Frog calls at 20-m increments from the recorder out to $160 \mathrm{~m}$ in each of the four cardinal directions on clear days when wind speed was $<10 \mathrm{~km} / \mathrm{h}$ and compared the number of broadcasted calls to the number of those calls that were distinguishable when we reviewed recordings made on automated recorders. We broadcasted Wood Frog calls at 67 (range $=64-71$ ) dB (measured $1 \mathrm{~m}$ from the source, as above) and compared the number of broadcasted calls detected with the number of broadcasted calls for each distance. To further assess sensitivity of automated recorders under field conditions, we also conducted 25 listening surveys when automated recorders were operating. We compared the number of calling frogs detected based on listening to recordings from each location to the direct counts made simultaneously by the observer at the same locations. We used Wilcoxon matched-pairs tests because our data were not normally distributed (Conover 1999).

\section{RESULTS}

Surveys with broadcasted calls - We detected $\geq 1$ anuran at 47 of the 155 potential breeding sites in our sample, and we detected both species at 6 sites. The mean number of Wood Frogs we detected pre-call broadcast ( $\bar{x}=0.82, S D=1.38$ ) increased significantly (Wilcoxon test; $Z=2.73, P=0.006$; Figure 1 ) to 1.24 ( $S D=1.51$ SD) post-broadcast, indicating that Wood Frogs responded to broadcasted conspecific calls. This difference was not the result of difference in background call rates between surveys where we broadcast Wood Frog calls $(\bar{x}=$ 
$0.82, S D=1.38)$ and surveys with null treatments $(\bar{x}=$ $0.54, \mathrm{SD}=0.93 \mathrm{SD})$ (Mann-Whitney U-test, adjusted $Z$ $=0.938, \mathrm{P}=0.348$; Figure 1).

There was no statistically significant difference in the number of detections of Boreal Chorus Frogs pre- $(\bar{x}=$ $0.89, \mathrm{SD}=1.37)$ and post-broadcast $(\overline{\mathrm{X}}=1.00, \mathrm{SD}=$ 1.53; Wilcoxon test; $Z \approx 0.001, P>0.90$ ) of conspecific calls. Nor was there a difference in the number of Boreal Chorus Frogs we detected during the initial 3-min listening period of surveys during which we broadcasted Boreal Chorus Frog calls $(\bar{x}=0.89, S D=1.37)$ and surveys with null treatment $(\bar{x}=1.06, S D=1.80$; Mann-Whitney $\mathrm{U}$ test; adjusted $\mathrm{Z}<0.001, \mathrm{P}>0.90)$.

There was no difference in the number of Wood Frogs detected in the 3-min periods before $(\bar{x}=0.54$, SD $=$ $0.93 \mathrm{SD})$ and after $(\bar{X}=0.63, \mathrm{SD}=1.13)$ null treatments (Wilcoxon test; $Z<0.001, P>0.9$ ). Similarly, there was no difference in the number of Boreal Chorus Frogs we detected in the 3 -min periods before ( $\bar{x}=1.06$, $\mathrm{SD}=1.80)$ and after $(\overline{\mathrm{X}}=1.13, \mathrm{SD}=1.74)$ null treatments (Wilcoxon test; $Z<0.001, P>0.9$ ). Therefore, differences in the number of frogs detected prior to and subsequent to call broadcasts were not the result of our presence at a site.

Comparison between audile surveys and automated recorders - We detected $100 \%$ of broadcasted Wood Frog calls up to $100 \mathrm{~m}$, but detection declined at distances beyond $100 \mathrm{~m}$ and we detected no call broadcasts at 160 $m$ (Figure 2). During simultaneous listening ( $\bar{x}=0.96$, $S D=1.27)$ and automated recording surveys $(\bar{x}=0.60$, $\mathrm{SD}=0.87)$ we registered significantly fewer Wood Frogs in automated recording surveys $(Z=2.07, P=0.038$; Figure 3 ). In contrast, the number of chorus frogs registered using automated recorders $(\bar{X}=1.72, S D=1.31)$ and detected by observers $(\bar{x}=1.44, S D=1.45)$ was not different $(Z=1.55, P>0.121)$.

\section{DISCUSSION}

Eliciting calls from non-calling anurans and use of automated recorders has been used to document species richness, but may also be useful for documenting abundance and therefore may increase survey efficiency (Penman et al. 2005). These techniques may be especially useful in locations where established survey protocols may be logistically difficult to employ. In the subarctic tundra landscape near Cape Churchill, logistical constraints include the lack of vehicle access, the expense of operating out of a remote research camp, the presence of dangerous wildlife, and a short anuran breeding season during periods with variable and often inclement weather. Under

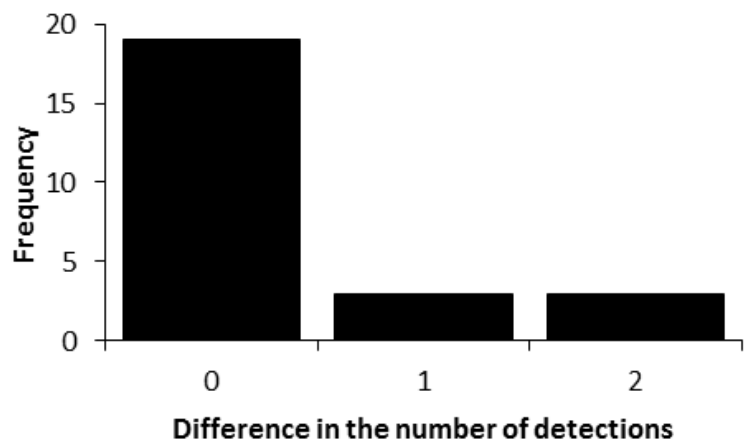

Figure 3. Difference in the number of Lithobates sylvaticus detections made by observers and using automated recorders during simultaneous surveys near Cape Churchill, Manitoba, 2006-2007. such conditions, methods that increase anuran detection can make surveys more feasible and efficient and facilitate studies of poorly-known populations.

Accurately estimating the number of calling anurans can be difficult when a chorus is large and individual calls are indistinguishable. For example, the North American Amphibian Monitoring Program (http://www.pwrc.usgs. gov/naamp/) uses species-specific call index values to categorize numbers of chorusing anurans when individuals are not distinguishable. In our study area, the density of anurans was relatively low (Reiter et al. 2008, present study), generally allowing us to distinguish individuals and precisely count the number of calling anurans. However, not all frogs present at a particular location call at any given time. When the goal of surveying anurans is to establish presence or estimate abundance, using methods that increase the likelihood that individuals will be detected during surveys is desirable. Eliciting responses using broadcasted calls is a common survey method that has been used extensively in bird surveys (e.g., Conway and Gibbs 2005, Andersen 2007). Broadcasting calls has elicited responses in some anuran species but not others (e.g., Zelick and Narins 1982) and it is therefore important to document which species respond to broadcasts to better construct efficient survey protocols. We found broadcasted calls of conspecifics slightly increased the number of Wood Frogs we detected per survey (Figure 1 ), but did not elicit calls from Boreal Chorus Frogs. Because of the biological importance of calls (Wells 1977; Wells and Greer 1981; Gerhardt 1982; Ryan 1985; Gerhardt et al. 2000), it is not surprising that an audile stimulus elicited a response from Wood Frogs. Why Boreal Chorus Frogs did not respond similarly is not clear, but not surprising. Lopez et al. (1988) found that some male Caribbean White-lipped Frogs (Leptodactylus albilabris) responded to call playbacks while others did not, and Zelick and Narins (1982) showed that call suppression can result from playbacks in Coquí (Eleutherodactylus coqui), presumably to avoid acoustic overlap with other individuals. Therefore, individual and species-specific differences in responses to call broadcasts are not new, and broadcasting calls is expected to work better for some species than for others. Our results suggest that broadcasting Wood Frog calls may increase detection, especially in areas were population densities are low.

Another way to enhance survey efficiency may be to use automated recorders that can collect information in the absence of observers. Although the automated recorders we used reliably registered anuran calls from as far as $100 \mathrm{~m}$ in any direction, our analysis of recordings did not always result in accurate assessment of frog abundance. The estimated number of calling Wood Frogs derived from automated recorders was lower than the number we detected when conducting simultaneous listening surveys, and this may be related to the characteristics of the calls and equipment. Automated recorders collapse the three-dimensional aural world detected by an observer into a single dimension. Using direction to identify the source of calls allows an observer to distinguish individuals, something that is difficult to do while listening to a recording. Wood Frogs call with considerable overlap among individuals, making it difficult to determine call orientation and accurately discern the number of frogs calling. The situation was different for Boreal Chorus Frogs, which usually call with considerably less overlap between individuals. Reduced call overlap likely allowed us to accurately assess the number of calling Boreal Chorus Frogs. 
Acevedo and Villanueva-Rivera (2006:211) found that recorders produced "better quantity and quality of data." Although our findings and those of Penman et al. (2005) are not completely consistent with those of Acevedo and Villanueva-Rivera (2006), we agree that recorders offer some benefits, not least of which is the ability to collect field data continuously. There are also disadvantages inherent in employing automated recorders (e.g., Dorcas et al. 2010), including, in our study, a large investment of time necessary to listen to recordings and our difficulty in accurately counting the number of calling Wood Frogs. Conversely, we were able to conduct surveys using automated records during times when it was not safe for observers to be in the field due to potential encounters with polar bears (e.g., during periods of low light). Based on our results, we believe that automated recorders would likely be effective in monitoring sites for presence or absence of calling frogs of many species, for detecting qualitative changes in chorus size, helping in studies of call seasonality, or facilitating documentation of longterm population trends. Their utility is likely to be especially high in remote locations with low-density populations, such as where we conducted our study, where the likelihood of observers hearing the calls of all species or individuals present during any short visit is low. Furthermore, automated recorders would likely be useful when estimating occupancy (e.g., MacKenzie et al. 2002) or presence of species in remote locations, especially if reviewing recordings could be automated. Overall, broadcasting calls and using automated recorders can lead to a decrease in required survey effort, potentially reduce costs (e.g., Acevedo and Villanueva-Rivera 2006), and for some species, may provide a means of surveying populations over a broad area efficiently.

\section{ACKNOWLEDGMENTS}

Funding and logistical support for this work was provided by Wapusk National Park of Canada, Texas Tech University, the U.S. Geological Survey, the Minnesota Cooperative Fish and Wildlife Research Unit, and the Eastern Prairie Population Canada Goose Committee of the Technical Section of the Mississippi Flyway Council. For assistance in the field we thank T. Bishop, M. Gillespie, C. Henneman, J. Huener, M. Jones, M. Reiter, J. Lawrence, B. Luebke, G. Lundie, S. Maxson, B. McCardle, M. Miller, B. Nack, B. Olson, M. Roell and W. Souer. M. Reiter also provided assistance with study area logistics and delineating our study areas. All work was conducted under Texas Tech University ACUC permit 06021-05 and Parks Canada research permit Wap-2005-518. This is manuscript T-9-1186 of the College of Agricultural Sciences and Natural Resource Management, Texas Tech University. Use of trade, firm, or product names is for descriptive purposes only and does not imply endorsement by the U.S. Government, the University of Minnesota, or Texas Tech University.

\section{LITERATURE CITED}

Acevedo, M.A., and L.J. Villanueva-Rivera. 2006. Using automated digital recording systems as effective tools for the monitoring of birds and amphibians. Wildlife Society Bulletin 34:211-214.

Andersen, D.E. 2007. Raptor survey techniques. Pages 89-100 in D.M. Bird and K. Bildstein, eds. Raptor research and management techniques (revised edition). Hancock House Publishers, Blaine, Washington, USA.

Brook, R. K. 2001. Structure and dynamics of the vegeta- tion of Wapusk National Park and the Cape Churchill Wildlife Management Area of Manitoba: community and landscape scales. Thesis, Natural Resources Institute, University of Manitoba, Winnipeg, Manitoba, Canada.

Conover, W.J. 1999. Practical nonparametric statistics. Third edition. John Wiley and Sons, New York, New York, USA.

Conway, C.J. and J.C. Simon. 2003. Comparison of detection probability associated with burrowing owl survey methods. Journal of Wildlife Management 67:501-511.

Conway, C.J. and J.P. Gibbs. 2005. Effectiveness of call-broadcast surveys for monitoring marsh birds. Auk 122:26-35.

Crouch, W.B., and P.W.C. Paton. 2000. Using egg mass counts to monitor Wood Frog populations. Wildlife Society Bulletin 28:895-901.

Didiuk, A. B., and D. H. Rusch. 1998. Movements of Canada goose broods near Cape Churchill, Manitoba. Pages 79-85 in D.H. Rusch, M.D. Samuel, D.D. Humburg, and B.D. Sullivan, editors. Biology and management of Canada geese. Proceedings of the International Canada Goose Symposium, Milwaukee, Wisconsin, USA.

Dorcas, M.E., S.J. Price, S.C. Walls, and W.J. Barichivich. 2010. Auditory monitoring of anuran populations. Pages 281-298 in C. K. Dodd Jr., editor. Ecology and conservation of amphibians: a handbook of techniques. Oxford University Press, New York, New York, USA.

Gerhardt, H.C. 1975. Sound pressure levels and radiation patterns of the vocalizations of some North American frogs and toads. Journal of Comparative Physiology 102:1-12.

Gerhardt, H.C. 1982. Sound pattern recognition in some North American tree frogs (Anuran: Hylidae). Implications for mate choice. American Zoologist 22:581595.

Gerhardt, H.C., S.D. Tanner, C.M. Corrigan, and H.C. Walton. 2000. Female preference functions based on call duration in the Gray Tree Frog (Hyla versicolor). Behavioral Ecology 11:663-669.

Heyer, W.R., M.A. Donnelly, R.W. McDiarmid, L.C. Hayek, and M.S. Foster. 1994. Measuring and monitoring biological diversity: standard methods for amphibians. Smithsonian Institution Press, Washington, D.C., USA.

Lopez, P.T., P.M. Narins, E.R. Lewis, and S.W. Moore. 1988. Acoustically induced call modification in the White-lipped Frog, Leptodactylus albilabris. Animal Behaviour 36:1295-1308.

Mannan, R.N. 2008. An assessment of survey methodology, calling activity, and habitat associations of Wood Frogs (Rana sylvatica) and Boreal Chorus Frogs (Pseudacris maculata) in a tundra biome. M.S. thesis, Texas Tech University, Lubbock, Texas, USA.

MacKenzie, D.I., J.D. Nichols, G.B. Lachman, S. Droege, J.A. Royle, and C.A. Langtimm. 2002. Estimating site occupancy rates when detection probabilities are less than one. Ecology 83:2248-2255.

Penman, T.D., F.L. Lemckert, and M.J. Mahony. 2005. A cost-benefit analysis of automated call recorders. Applied Herpetology 2:389-400.

Peterson, C.R. and M.E. Dorcas. 1992. The use of automated data-acquisition techniques in monitoring amphibian and reptile populations. Pp. 369-378 in D.R. McCullough, and V. Barrett, eds. Wildlife 2001: 
Populations. Elsevier, Applied Science, London, U.K. Peterson, C.R. and M.E. Dorcas. 1994. Automated data acquisition. Pp. 47-57 in W.R. Heyer,, M.A. Donnelly, R.W. McDiarmid, L.C. Hayek, M.S. Foster, eds. Measuring and monitoring biological diversity: Standard methods for amphibians. Smithsonian Institution Press, Washington, DC, USA.

Reiter, M.E., D.E. Andersen, and C.W. Boal. 2008. Anurans in a subarctic landscape near Cape Churchill, Manitoba. Canadian Field-Naturalist 122:129-137.

Ryan, M. 1985: The Túngara Frog. The University of Chicago Press, Chicago, Illinois, USA.
Shirose, L.J., C.A. Bishop, D.M. Green, C.J. MacDonald, R.J. Brooks, and N.J. Helferty. 1997. Validation tests of an amphibian call count survey technique in Ontario, Canada. Herpetologica 53:312-320.

Wells, K.D. 1977. The social behavior of anuran amphibians. Animal Behaviour 25:666-693.

Wells, K.D. and B.J. Greer. 1981. Vocal responses to conspecific calls in a neotropical Hylid Frog, Hyla ebraccata. Copeia 1981:615-624.

Zelick, R.D., and P.M. Narins. 1982. Analysis of acoustically evoked call suppression behaviour in a neotropical treefrog. Animal Behaviour 30:728-733. 